\title{
Calibraciones de espectrofotometría de reflectancia en el infrarrojo cercano para carbohidratos en pasto Urochloa
}

\section{Near-Infrared reflectance spectroscopy calibrations for carbohydrate in Urochloa}

\author{
grass
}

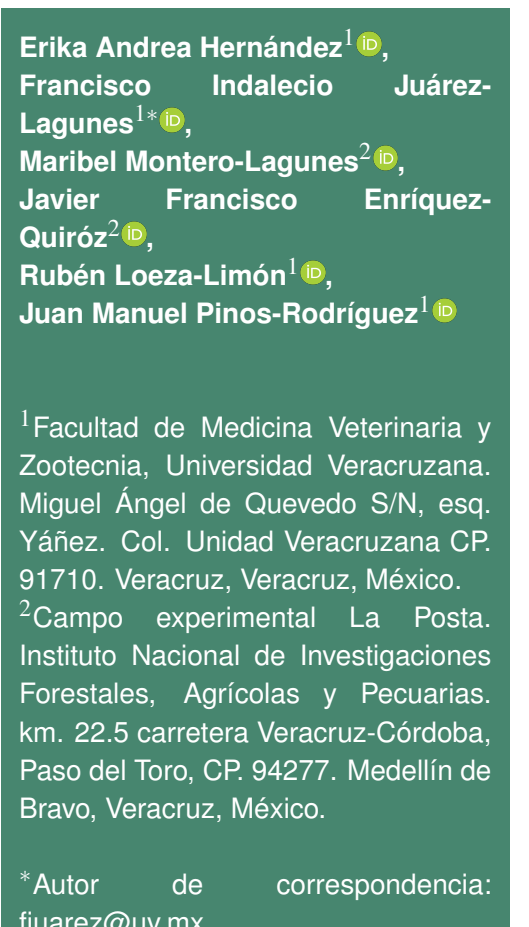

fjuarez@uv.mx

Artículo científico

Recibido: 22 de febrero 2021

Aceptado: 01 de octubre 2021

Como citar: Hernández EA, JuárezLagunes FI, Montero-Lagunes M, Enríquez-Quiróz JF, Loeza-Limón R, Pinos-Rodríguez JM (2021) Calibraciones de espectrofotometría de reflectancia en el infrarrojo cercano para carbohidratos en pasto Urochloa. Ecosistemas y Recursos Agropecuarios Núm. Esp. II: e2874. DOI: 10.19136/era.a8nll.2874
RESUMEN. El objetivo fue desarrollar modelos de calibración de espectroscopia de reflectancia en el infrarrojo cercano (NIRS) para estimar el contenido de las fracciones de fibra y de carbohidratos en pastos Urochloa sp. en la zona tropical de México. Ciento ochenta y nueve muestras de tres híbridos de Urochloa fueron cosechadas cada 35 días durante un año, las cuales se analizaron por química húmeda para las fracciones de Fibra Detergente Neutra (FDN), Proteína Insoluble en Detergente Neutro (PIDN), Fibra Detergente Ácida (FDA), Proteína Insoluble en Detergente Ácido (PIDA) y Lignina (LIG), También se estimaron los carbohidratos totales (CT), carbohidratos fibrosos (CF), carbohidratos no fibrosos (CNF) y carbohidratos indigestibles $(\mathrm{Cl})$. Los modelos de calibración y de validación se evaluaron por los respectivos Coeficientes de Determinación $\left(R^{2}\right)$; Errores Estándar de Validación Cruzada (EEVC); y la desviación residual de la predicción (DRP). Las calibraciones obtenidas presentaron un adecuado ajuste y eficiencia predictiva mediante espectrofotometría NIR para FDN (DRP 2.4) y CT (DRP 2.5) lo que demuestra confiabilidad y capacidad de predicción para estas fracciones. Sin embargo, LIG, CF, CNF y Cl requieren más observaciones para acrecentar su confiabilidad.

Palabras clave: Brachiaria, CNCPS, composición química, FDN, NIRS.

ABSTRACT. The goal was to develop near infrared reflectance spectroscopy (NIRS) calibration models to estimate fiber and carbohydrate fractions content in Urochloa grasses grown in tropical Mexico. One hundred and eighty-nine samples of three Urochloa hybrids were cut at 35 days of regrowth for a year. Grasses were wet-chemistry analyzed for neutral detergent fiber (NDF), neutral detergent insoluble protein (NDIP), acid detergent fiber (ADF), acid detergent insoluble crude protein (ADIP), and lignin (LIG). Also, estimations of total carbohydrates (TC), fibrous carbohydrates (FC), non-fibrous carbohydrates (NFC), and undigestible carbohydrates (UC) were computed. Calibration and validation models were evaluated by their corresponding determination coefficients $\left(R^{2}\right)$; cross validation standard errors (SECV); and the residual prediction of the deviation (RPD). The NIRS calibration models developed achieved adequate adjustment and predictive efficiency for NDF (RPD 2.4) and TC (RPD 2.5) showing reliability to predict the fiber and carbohydrate fractions of Urochloa grasses. However, LIG, FC, NFC and UC calibration models require a larger number of samples to increase the prediction accuracy.

Key words: Brachiaria, chemical composition, CNCPS, NDF, NIRS. 


\section{INTRODUCCIÓN}

Los recursos forrajeros son importantes en la nutrición de rumiantes, proveen más del $90 \%$ de la energía consumida por éstos en todo el mundo (Wilkins 2000). Los pastos están compuestos principalmente por polisacáridos, que sirven como fuente primaria de energía para los rumiantes al ser fermentados por los microorganismos del rumen (Segura et al. 2007). Los carbohidratos pueden clasificarse como carbohidratos fibrosos (CF), representados por celulosa y hemicelulosa, y no fibrosos (CNF), representados por ácidos orgánicos, azúcares solubles en agua (mono y disacáridos), almidón y fibra soluble (principalmente pectinas) (Cabral et al. 2000). El CNCPS - The Cornell Net Carbohydrate and Protein System - con base en la clasificación de los carbohidratos según su composición y tasas de digestión, dinamiza la digestión de éstos para la obtención de energía. De tal forma que: la fracción A, representa a los ácidos orgánicos y azúcares solubles, y que se fermentan fácilmente en el rumen; la fracción $B_{1}$, comprende almidón y pectina, y que tienen velocidades intermedias de digestión; la fracción $B_{2}$, que es la pared celular potencialmente digestible y corresponde a la fracción lenta; y fracción C, representada por la porción indigerible de la fibra a lo largo del tubo gastrointestinal (Sniffen et al. 1992).

El conocimiento de las fracciones de fibra y de carbohidratos de los forrajes tropicales es una necesidad en los sistemas de producción bovina en pastoreo para poder utilizar el CNCPS en la toma de decisiones de manejo nutricional (Sniffen et al. 1992). La creciente demanda de tecnologías sencillas, rápidas, con alta repetibilidad, reproducibilidad y precisión para caracterizar la fibra y los carbohidratos de los pastos ha hecho que la espectroscopia de reflectancia en el infrarrojo cercano (NIRS por sus siglas en inglés) gane espacio en estas evaluaciones (Li et al. 2016). Esta técnica sobresale en relación con los análisis convencionales, ya que estos son generalmente laboriosos, lentos y costosos, además de generar residuos con un gran potencial de contaminación ambiental (Lobos et al. 2013, Gonçalves et al. 2018).
Se han realizado varios estudios en el desarrollo y validación de ecuaciones NIRS para predecir la composición química de forrajes tropicales. Al respecto, Mazabel et al. (2020) desarrollaron modelos de calibración en la especie forrajera Brachiara humidicola (syn. Urochloa humidicola) para determinar las concentraciones de fibra detergente neutra (FDN) y fibra detergente ácido (FDA). De manera similar, Freitas et al. (2016) desarrollaron modelos para Lignina (LIG) en la misma especie forrajera. Por otra parte, Vásquez et al. (2004) desarrollaron modelos para carbohidratos no fibrosos (CNF) en el pasto Guinea (Megathyrsus maximus) y Nie et al. (2009) en forrajes templados. Estos estudios demuestran el potencial de los modelos de calibración NIRS para evaluar de manera eficiente los parámetros nutricionales de forrajes tropicales. Sin embargo, esta técnica no ha sido suficientemente utilizada para la estimación de las fracciones de fibra y carbohidratos, de acuerdo con la estructura del CNCPS, en pastos tropicales de México. Por lo anterior el objetivo fue desarrollar los modelos de calibración NIRS para las fracciones de fibra y de carbohidratos con base en la estructura del CNCPS en muestras de tres cultivares del género Urochloa para la zona tropical de México.

\section{MATERIALES Y MÉTODOS}

\section{Área de estudio}

El estudio se realizó en el Campo Experimental La Posta del INIFAP. El clima de la región es Aw caliente subhúmedo (García 1987) con temperatura y precipitación promedio anual de $25^{\circ} \mathrm{C}$ y $1380 \mathrm{~mm}$ respectivamente. La altura sobre el nivel del mar es de $16 \mathrm{~m}$. La posición geoespacial comprende los paralelos $19^{\circ} 02^{\prime} \mathrm{LN}$ y $96^{\circ} 08^{\prime} \mathrm{LO}$. El suelo se clasifica como Vertisol, de textura arenosa con más de $18.80 \%$ de arcilla, $2.61 \%$ de materia orgánica, y $\mathrm{pH}$ de 6.1 .

\section{Muestras, diseño experimental y tratamiento}

Se establecieron 18 parcelas $(3 * 2 \mathrm{~m}$ ) con área de muestreo de $1 \mathrm{~m}^{2}$ cada una. Los muestreos se realizaron en el año 2013 cada 35 días a una altura 
de planta de $15 \mathrm{~cm}$. Se incorporó variación para los efectos fijos de especie, y fertilización con riego con el fin de tener mayor amplitud en los espectros NIR y que pudieran elaborarse ecuaciones de predicción para esas condiciones. Las especies de Urochloa utilizadas fueron: Dictyoneura (Urochloa humidicola); Insurgente (Urochloa brizantha); y Mulato I (Urochloa ruziziensis $\mathrm{X} U$. brizantha). Para cada especie se establecieron seis parcelas: Tres parcelas se fertilizaron con $\mathrm{N}$ y tres no. Las parcelas fertilizadas se fertilizaron con la fórmula 150-60-00, $\mathrm{kg} \mathrm{ha}^{-1} \mathrm{año}^{-1}$ de $\mathrm{N}, \mathrm{P}$ y K , de los cuales se proporcionaron $75 \mathrm{~kg} \mathrm{ha}^{-1}$ de urea al corte de uniformización, posteriormente $25 \mathrm{~kg}$ de urea después de cada corte, mientras que el fósforo se aplicó en una sola ocasión al inicio del experimento. Se suministró riego por goteo durante las épocas de invierno y secas (de diciembre a mayo) solo a las parcelas fertilizadas. En total se analizaron 189 muestras del género Urochloa, de las cuales 126 fueron para la calibración de las ecuaciones, y 63 para la validación externa.

\section{Análisis de laboratorio}

Las muestras de forraje colectadas fueron secadas en horno de aire forzado a $55^{\circ} \mathrm{C}$ hasta peso constante y molidas en un molino Wiley (Model 4, Arthur H. Thomas Co. Philadelphia, PA) con malla de $1 \mathrm{~mm}$. Se determinó Fibra Detergente Neutro (FDN), Fibra Detergente Ácido (FDA) y Lignina (LIG) (Van Soest et al. 1991). Además, se hicieron las determinaciones de proteína insoluble en FDN (PIDN) y FDA (PIDA) (Licitra et al. 1996). Las fracciones de fibra y de carbohidratos fueron determinadas como se muestran en la Figura 1 y Tabla 1.

\section{Obtención espectros NIRS}

Una vez conocidas las fracciones de fibra y de carbohidratos de las Urochloas, éstas fueron escaneadas en el equipo Thermo Scientifc Nicolet ${ }^{T M}$ 6700 , en un rango de longitudes de onda de 1000 a $2500 \mathrm{~nm}$ de reflectancia. El proceso de escaneo se realizó colocando la muestra sobre un contenedor con ventana de cuarzo, el cual es inactivo a las ondas electromagnéticas del infrarrojo, posteriormente el contenedor se colocó sobre la esfera de integración del espectrofotómetro y se puso a girar durante el proceso de escaneo de la muestra. Para la obtención de los espectros se utilizó el software OMNIC versión 2008.

\section{Obtención de los Modelos de Calibración}

Los espectros NIRS se correlacionaron con los valores para las fracciones de fibra y de carbohidratos descritas en la Tabla 1 para obtener los modelos de calibración. Para cada parámetro se desarrolló un modelo individual. Se aplicó la variable normal estándar (SNV) a todos los espectros, con el fin de eliminar los efectos de la dispersión de los datos espectrales para poder observar las diferencias químicas y limitar la región utilizada para así corregir las longitudes de trayectoria si en uno o en ambos extremos de los datos espectrales se presentaban numerosos ruidos. Por otra parte, se realizó la identificación de un conjunto de valores atípicos, outliers, los cuales fueron analizados para su eliminación cuando fuera necesario. También, se utilizaron tratamientos matemáticos (Tabla 2) basados en derivadas de primer orden, para optimizar la extracción de información útil de los espectros. Finalmente se aplicó el modelo de regresión de mínimos cuadrados parciales (PLS) para la calibración de los espectros. La aplicación del tratamiento matemático previo a los espectros, la evaluación de valores atípicos y la calibración se realizaron utilizando el software de quimiometría $T Q$ Analyst V8.

\section{Selección de los modelos de calibración}

El modelo de calibración para cada parámetro se seleccionó teniendo en cuenta la relación entre el coeficiente de determinación $\left(R^{2}\right)$ más alto y el error estándar de validación cruzada de la calibración (EEVCc) más bajos (Alomar et al. 2003, Vásquez et al. 2004). La bondad y la precisión de los modelos de calibración se probaron utilizando la desviación residual de la predicción (DRPc) calculada como la relación entre la desviación estándar (DEc) de los valores de referencia y el error estándar de validación cruzada de la calibración (EEVCc), algunos autores indican que esta ecuación tiene alta capacidad de predicción (Arana et al. 2005, Cozzolino y Moron 


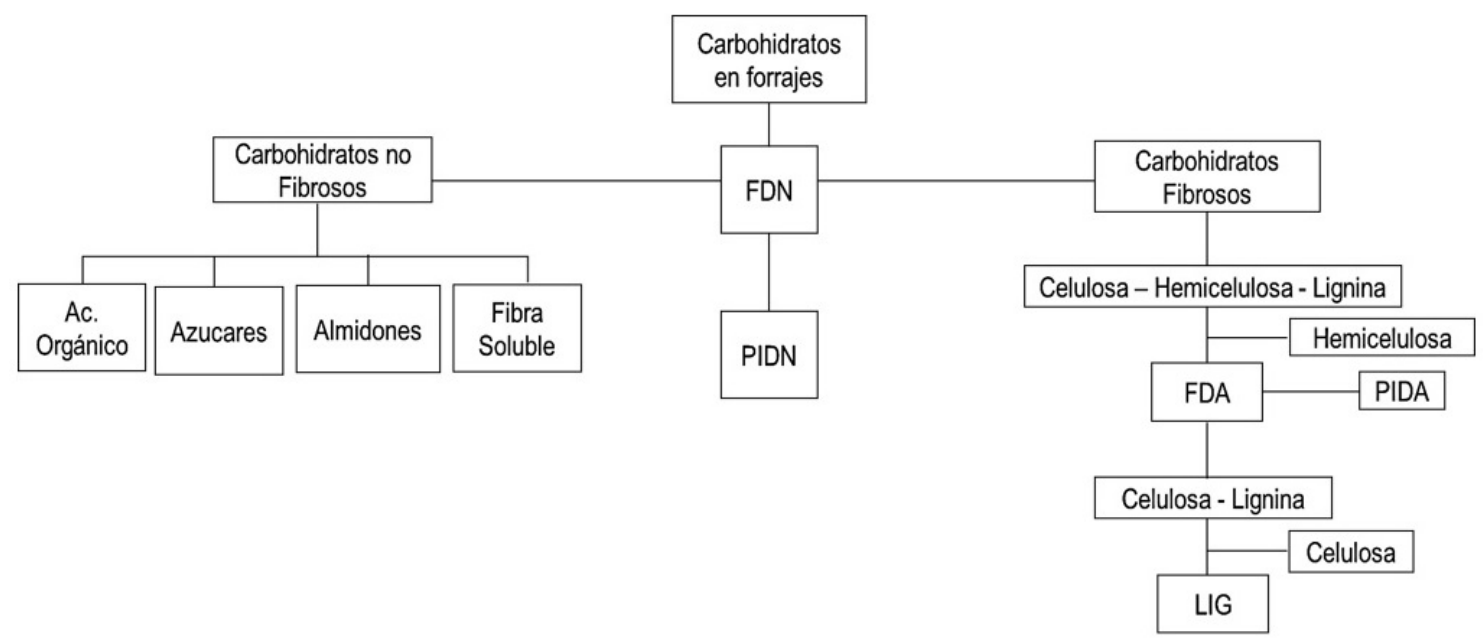

Figura 1. Fracciones de fibra y de carbohidratos en forrajes. Adaptado de Hall et al. 1999.

Tabla 1. Estimación de las fracciones de fibra y de carbohidratos.

\begin{tabular}{ccc}
\hline Fracción & Descripción & Ecuación \\
\hline \multicolumn{3}{c}{ Fracciones de Fibra } \\
FDN, LIG & Van Soest et al. (1991) \\
FI & Fibra Indigestible & Fracciones de Carbohidratos \\
& Carbohidratos Totales & $100-$ PC - EE - CEN - LIG \\
CT & Carbohidratos Fibroso & FDN - PIDN - LIG \\
CF & Carbohidratos No Fibrosos & $100-$ PC - EE - CEN - (FDN -PIDN) \\
CNF & Carbohidratos Fibrosos Indigestibles & FI - LIG \\
CFI & Carbohidratos Fibrosos Digestibles & FDN - PIDN - FI \\
CFD & Carb /
\end{tabular}

FDN: fibra detergente neutro, LIG: lignina, PC: proteína cruda, EE: extracto etéreo, CEN: cenizas, PIDN: proteína insoluble en detergente neutro.

2004, Molano et al. 2016, Shenderey et al. 2010).

Tabla 2. Tratamiento matemático de la calibración de las fracciones de fibra y de carbohidratos.

\begin{tabular}{cccc}
\hline Parámetro & MC & FPLS & Tratamiento matemático \\
\hline \multicolumn{5}{c}{ Fracciones de Fibra } \\
FDN & 108 & 8 & $1,3,1$ \\
LIG & 101 & 10 & $1,3,1$ \\
FI & 104 & 7 & $1,3,1$ \\
\multicolumn{5}{c}{ Fracciones de Carbohidratos } \\
CT & 109 & 7 & $1,3,1$ \\
CF & 103 & 5 & $1,3,1$ \\
CNF & 106 & 6 & $1,3,1$ \\
CFI & 102 & 5 & $1,3,1$ \\
CFD & 106 & 4 & $1,3,1$
\end{tabular}

FDN: fibra detergente neutro, LIG: lignina, FI: fibra indigestible, CT: Carbohidratos totales, CF: carbohidratos fibrosos, CNF: carbohidratos no fibrosos, CFI: carbohidratos fibrosos indigestibles, CFD: carbohidratos fibrosos digestibles, MC: muestras para calibración, FPLS: número de factores, PLS, 1: $1^{s t}$, 3: polinomial, 1: smooth.

\section{Validación de los Modelos de Calibración}

Seleccionados los modelos de calibración, se realizó la validación externa con el fin de determinar su precisión mediante el coeficiente de determinación $\left(R^{2}\right)$, el error estándar de validación cruzada de la predicción (EEVCp) y la DRPp de la predicción (DEp/EEVCp).

\section{Análisis estadístico}

Los datos se sometieron a un análisis de estadísticas descriptivas (media, desviación estándar $(\mathrm{DE})$, coeficiente de variación $(\mathrm{CV})$, valor máximo (max), y valor mínimo (min)) con MINITAB Versión 15. Los modelos seleccionados fueron evaluados mediante análisis de regresión lineal y el Cuadrado Medio del Error de Predicción (CMEP) el cual evalúa el rendimiento de la regresión del modelo y expresa las diferencias entre el valor predicho y los valores de 
referencia medidos (Lobos et al. 2013).

\section{RESULTADOS}

La estadística descriptiva del contenido de FDN, LIG, fibra indigestible $(\mathrm{FI})$ y de las fracciones de carbohidratos CT, CF, CNF y CFD de los forrajes utilizados para la calibración y validación de las ecuaciones se presentan en la Tabla 3. Las medidas de dispersión de los análisis químicos muestran alta variabilidad entre los parámetros evaluados en los cultivares de Urochloa, lo que indica amplia distribución de las muestras, requisito necesario para el desarrollo de curvas de predicción confiables utilizando el equipo NIRS.

Tabla 3. Estadística descriptiva de la composición química de las fracciones de fibra y de carbohidratos (\% MS).

\begin{tabular}{ccccccc}
\hline Parámetro & No. & Media & DE & CV & Max & Min \\
\hline \multicolumn{7}{c}{ Fracciones de Fibra } \\
FDN & 189 & 64.9 & 5.31 & 8.2 & 77.4 & 52.4 \\
LIG & 189 & 5.9 & 1.28 & 21.7 & 10.5 & 1.8 \\
FI & 189 & 14.1 & 3.06 & 21.7 & 25.1 & 4.4 \\
Fracciones de Carbohidratos \\
CT & 189 & 70.9 & 5.02 & 7.1 & 80.3 & 60.0 \\
CF & 189 & 55.5 & 5.84 & 10.5 & 69.2 & 41.5 \\
CNF & 189 & 15.3 & 4.43 & 28.8 & 24.7 & 4.1 \\
CFI & 189 & 8.2 & 1.79 & 21.7 & 14.6 & 2.6 \\
CFD & 189 & 47.3 & 6.73 & 14.2 & 62.4 & 26.9 \\
\hline
\end{tabular}

FDN: fibra detergente neutro, LIG: lignina, FI: fibra indigestible, CT: Carbohidratos totales, CF: carbohidratos fibrosos, CNF: carbohidratos no fibrosos, CFI: carbohidratos fibrosos indigestibles, CFD: carbohidratos fibrosos digestibles, No.: Número de muestras; DE: desviación estándar; $\mathrm{CV}$ : coeficiente de variación, Max: máximo, Min: Mínimo.

La Tabla 4 muestra los parámetros estadísticos de desempeño en los modelos de calibración seleccionados y su validación. Los resultados destacan la eficiencia predictiva de los modelos de calibración al obtener EEVCc bajos. El coeficiente de determinación que indica el ajuste del modelo tuvo una $R^{2}$ c entre 0.65 y 0.94 y la DRPc entre 3.2 y 1.3 , lo cual indica que la predicción de algunos de los modelos seleccionados es alta y de otros no. Seleccionados los modelos de calibración con mejor desempeño, se realizaron las respectivas validaciones utilizando 63 muestras de los colectivos de calibración (Tabla 4). La variación explicada en los modelos de validación $\left(R^{2} v\right)$ entre la predicción con la técnica NIRS y los datos de laboratorio, presentaron valores de 0.92 a 0.45 , con excepción de carbohidratos fibrosos indigestibles (CFI) que presentaron $R^{2}=0.32$. Con respecto al EEVCp los carbohidratos fibrosos digestibles (CFD) tendrían la mayor variación de predicción al obtener un resultado de 4.1. Los resultados de DRPp fueron $\geq 2.8$ para FDN y carbohidratos totales (CT). Fibra indigestible (FI) obtuvo el menor valor con 1.0 (Tabla 4).

\section{DISCUSIÓN}

\section{Composición química de las fracciones de fibra y de carbohidratos}

Se observó amplia variación en las concentraciones de los diferentes parámetros analizados en el laboratorio para las Urochloa evaluadas. Los coeficientes de variación (CV) y la desviación estándar (DE) presentaron oscilación entre 7.1 a $28.8 \%$ y de 1.28 a $6.73 \%$, respectivamente (Tabla 3 ). Los resultados para FDN son similares a los reportados por Vásquez et al. (2004) en la especie Megathyrsus maximus que tuvo CV y DE de 7.26 y $4.77 \%$, respectivamente; mientras que Mazabel et al. (2020) para Urochloa humidicola reporta valores de $7.45 \%$ y $4.83 \%$ para el CV y la DE. Para LIG los resultados estuvieron por debajo de lo reportado por Parrini et al. (2018) con un CV y DE de 41.90 y $2.39 \%$, respectivamente. La diferencia puede ser por el número de muestras analizadas, las cuales pueden afectar el resultado esperado de las ecuaciones de calibración (Pasquini 2003). Al repecto, Valenciaga y Saliba (2006) indican que mientras mayor es el número de muestras, mayor precisión se logra en la determinación y, por tanto, en la calibración. Las fracciones de carbohidratos han sido poco analizadas por medio de espectros NIR en pastos tropicales. Sobre esto Nie et al. 2009 evaluaron la factibilidad de usar NIRS para predecir concentraciones de ácidos orgánicos, almidón, azúcares, fibra soluble en detergente neutro y carbohidratos solubles en detergente en Alfalfa y Timothy en Canadá.

Respecto a los modelos de calibración para FDN, la cual es considerada en los espectros NIRS 
Tabla 4. Calibración y Validación de las fracciones de fibra y de carbohidratos (\% MS)

\begin{tabular}{|c|c|c|c|c|c|c|c|c|c|c|c|c|}
\hline \multirow[b]{2}{*}{ Parámetro } & \multicolumn{5}{|c|}{ Calibración } & \multicolumn{7}{|c|}{ Validación } \\
\hline & No. & Media & $\mathrm{DEc}$ & EEVCc & $\mathrm{R}^{2} \mathrm{c}$ & DRPc & No. & Media & DEp & EEVCp & $R^{2} v$ & DRPp \\
\hline \multicolumn{13}{|c|}{ Fracciones de Fibra } \\
\hline FDN & 108 & 64.73 & 5.31 & 1.65 & 0.82 & 3.2 & 48 & 64.8 & 4.80 & 1.43 & 0.91 & 3.4 \\
\hline LIG & 101 & 5.87 & 0.95 & 0.60 & 0.94 & 1.6 & 49 & 5.97 & 0.73 & 0.61 & 0.45 & 1.2 \\
\hline $\mathrm{FI}$ & 104 & 14.18 & 2.33 & 1.78 & 0.72 & 1.3 & 54 & 14.4 & 1.74 & 1.76 & 0.48 & 1.0 \\
\hline \multicolumn{13}{|c|}{ Fracciones de Carbohidratos } \\
\hline CT & 109 & 70.4 & 5.14 & 1.92 & 0.73 & 2.7 & 59 & 70.6 & 4.77 & 1.69 & 0.91 & 2.8 \\
\hline CF & 103 & 55.5 & 5.60 & 2.11 & 0.71 & 2.7 & 43 & 55.3 & 5.09 & 3.55 & 0.92 & 1.4 \\
\hline CNF & 106 & 15.3 & 4.26 & 1.93 & 0.66 & 2.2 & 45 & 14.8 & 4.22 & 1.94 & 0.84 & 2.2 \\
\hline CFI & 102 & 8.19 & 1.31 & 1.05 & 0.65 & 1.2 & 59 & 8.30 & 1.01 & 0.22 & 0.32 & 4.6 \\
\hline CFD & 106 & 47.6 & 6.43 & 3.08 & 0.67 & 2.1 & 39 & 47.9 & 5.51 & 4.08 & 0.89 & 1.4 \\
\hline $\begin{array}{l}\text { FDN: fibra } \\
\text { CNF: carb } \\
\text { número de } \\
\text { coeficiente }\end{array}$ & ter & & LIG & & & & & & & & iidrato & $\begin{array}{l}\text { rosos, } \\
\text { s, No: } \\
\text { n, } \text { R }^{2}\end{array}$ \\
\hline
\end{tabular}

debido a las adsorciones - $\mathrm{CH}$ y $-\mathrm{OH}$, el $\mathrm{R}^{2}$ y EEVCc fue de 0.82 y 1.65 , respectivamente (Tabla 4). Valores que son similares a lo reportado por Freitas et al. (2016) con valores de $R^{2}$ de 0.89 y EEVCc de 0.13 para FDN en muestras de Urochola humidicola en Brasil. Mientras que Monrroy et al. (2017) observaron valores de $R^{2}$ de 0.87 y EEVCc de 1.85. en la especie Urochloa sp. Por el contrario Danieli et al. (2004) reportaron $\mathrm{R}^{2}$ de 0.94 y EEVC 2.17 en muestras de gramíneas y leguminosas, por lo que son superiores a los hallados en el presente estudio, pero posiblemente se pueden deber a que los modelos fueron desarrollados con diferentes especies de pastos y en condiciones ambientales diferentes.

La lignina presento el $R^{2}$ más alto con 0.94 y EEVCc de 0.60 (Tabla 4). Estos resultados pueden ser comparados con Freitas et al. (2016) y Fekadu et al. (2010) los cuales reportan $R^{2}$ de 0.89 y 0.77 , EEVC de 0.11 y 0.77 . Al respecto, Fernández et al. (2006) indican que en la región del infrarrojo es difícil determinar entidades complejas formadas por mezclas de varios compuestos, como el caso de los constituyentes de la pared celular vegetal. En esta región del espectro se miden, principalmente, las vibraciones de los enlaces químicos. En estos, el hidrógeno se une con átomos de nitrógeno, oxígeno y carbono, y probablemente, la celulosa, hemicelulosas, pectina y otros constituyentes de la pared celular absorben longitudes de onda similares, por lo que las bandas se pueden superponer y no permite cuan- tificarlos adecuadamente.

Aunque cenizas y extracto etéreo se analizaron químicamente, no se utilizaron en este trabajo debido a que la determinación de cenizas mediante NIRS podría presentar alteraciones, ya que puede verse afectada por la ausencia de adsorción en la región del infrarrojo cercano para los minerales. Al respecto, Parrini et al. (2018) indican que es posible la estimación del contenido de cenizas en forrajes probablemente por el conjunto que se forma entre éstas con compuestos orgánicos. Para extracto etéreo, el cual es posible identificar mediante NIRS por la adsorción alifática- $\mathrm{CH}$, la predicción de lípidos es poco frecuente debido a la baja concentración en el tejido y a los estrechos rangos en las plantas forrajeras (Parrini et al. 2018). Sobre lo mismo Roberts et al. (2003) reportan que la calibración de lípidos en diferentes muestras de forraje siempre presenta un resultado más bajo, mientras que Stuth et al. (2003) sugirieren resultados mixtos en las medidas de forraje debido a la baja varianza relacionada con la pequeña concentración.

Las fracciones de carbohidratos son componentes importantes en la calidad nutricional de los pastos tropicales (Van Soest 1994). El CNCPS las obtiene por ecuaciones sumativas a partir de la composición química (Lanzas et al. 2007). Algunos de los componentes químicos, como las cenizas y el extracto etéreo, necesarios para obtener los carbohidratos totales (CT), son pobremente predichos por 
las calibraciones NIRS (Parrini et al. 2018, Nie et al. 2009, Roberts et al. 2003, Stuth et al. 2003). Esta limitación se puede solventar estimando directamente los CT y las otras fracciones de carbohidratos con la tecnología NIRS. De acuerdo con la Tabla 2, el 70.9\% de la materia seca (MS) de los Urochloa estudiados son $\mathrm{CT}$, de éstos, $55.5 \%$ son $\mathrm{CF}$, siendo esta fracción potencialmente digestible (CFD) hasta $47.3 \%$. Los CNF se encuentran en menor proporción con 15.3\% de la MS, estas fracciones de carbohidratos coinciden con las estimadas por Hernández et al. (2020) utilizando la técnica de producción de gas in vitro. A partir de las calibraciones NIRS obtenidas (Tabla 4) los resultados de DRPc fueron de 2.7, 2.7, 2.2 y 2.1 para CT, CF, CNF, y CFD respectivamente. Calibraciones consideradas como justas para predecir estas fracciones de carbohidratos (Mazabel et al. 2020). La fracción CFI requiere de mayor número de observaciones para una predicción confiable. Diversas investigaciones mencionan que existe información de calibración NIRS prometedora para carbohidratos solubles en forrajes (Nie et al. 2009, Lobos et al. 2019, Piaskowski et al. 2016, Ramírez et al. 2015, Alomar et al. (2009). Pero para carbohidratos estructurales, que son los más importantes en pastos tropicales para rumiantes, no se encontró información en la literatura.

El índice DRP (relación DEc/EEVCc), mide la fuerza de la relación entre los valores de un componente y el error de los resultados predichos por NIRS (Williams 2014), el valor fue mayor o igual a 3 para FDN con 3.2, lo que indica que el modelo tiene un poder de predicción alto, al ser este el valor recomendado (Lobos et al. 2019, Molano et al. 2016). Por otra parte, Lobos et al. (2019) indican que un valor de DRP por debajo de 2.4 caracteriza un modelo deficiente, mientras que los valores de DRP de aproximadamente 2.5 a 3.0 podrían utilizarse para fines de detección. Pero Mazabel et al. (2020), sugieren que una DRP por debajo de 1.9 no es recomendable para las pruebas de forrajes, entre 2.0 a 2.4 son suficientes para una selección aproximada, entre 2.5 y 2.9 ofrecen un potencial de selección justo y superiores a 3.0 son altamente predictivos. Por lo anterior, la ecuación de FDN pueden ser utilizada para hacer predicciones cuantitativas confiables. Las ecuaciones de CT y CF permiten hacer predicciones justas, mientras que para CNF y CFD deben utilizarse con precaución. La DRP más baja la presentaron los parámetros de LIG, FI y CFI con valores menores a 1.6 (Tabla 4), por lo que no es recomiendan utilizar estas últimas ecuaciones hasta ajustarlas y validarlas.

En la validación externa de los modelos de calibración (Tabla 4) los únicos modelos que presentaron $R^{2}>0.90$, fueron FDN, CT y $C F$, aunque solo FDN y CT mostraron DRPp altos (3.4 y 2.8, respectivamente) lo que indica excelente precisión de predicción. Los CFD y los CNF obtuvieron $\mathrm{R}^{2} \geq$ 0.80 (Tabla 4) pero solo CNF obtuvo PRDp mayor a 2.0 que le permite hacer predicciones justas. Las calibraciones para LIG, FI, CF, CFI y CFD fallaron en la precisión de la predicción de acuerdo con los criterios de recomendación sugeridos por Shenk y Westerhaus (1996) y Williams (2004). Sobre lo mismo Nie et al. (2009) encontró el mismo problema de baja predicción en varias fracciones de carbohidratos estudiando Alfalfa y Timothy por separado. Pero cuando se combinaron las dos especies de forrajes las calibraciones de predicción mejoraron considerablemente. Quizás con pastos tropicales incorporando otras especies para generar calibraciones más precisas para fracciones de carbohidratos se puedan lograr resultados similares.

\section{CONCLUSIONES}

Los resultados muestran que las ecuaciones NIRS desarrolladas para las fracciones de fibra y carbohidratos como FDN y CT en la especie Urochloa spp tienen un ajuste adecuado y eficiencia predictiva para estimar los valores de estos componentes.

\section{LITERATURA CITADA}

Alomar D, Gallo C, Castañeda M, Fuchslocher R (2003) Chemical and discriminant analysis of bovine meat by 
near infrared reflectance spectroscopy (NIRS). Meat Science 63: 441-450.

Alomar D, Fuchslocher R, Cuevas J, Mardones R, Cuevas E (2009) Prediction of the composition of fresh pastures by near infrared reflectance or interactance-reflectance spectroscopy. Chilena Journal of Agricultural Research 69: 198-206.

Arana I, Jarén C, Arazuri S (2005) Maturity, variety and origin determination in white grapes (Vitis vinifera L.) using near infrared reflectance technology. Journal of Near Infrared Spectroscopy 13: 349-357.

Cabral LS, Valadares FSC, Malafaia PAM, Lana RP, Coelho SJF, Vieira RAM, Pereira ES (2000) Frações de carboidratos de volumosos tropicais e suas taxas de degradação estimadas através da técnica de produção de gases. Revista Brasileira de Zootecnia 29: 2087-2098.

Cozzolino D, Moron A (2004) Exploring the use of near infrared reflectance spectroscopy (NIRS) to predict trace minerals in legumes. Animal Feed Science and Technology 111: 161-173.

Danieli PP, Carlini P, Bernabucci U, Ronchi B (2004) Quality evaluation of regional forage resources by means of near infrared reflectance spectroscopy. Italian Journal of Animal Science 3: 363-376.

Fekadu D, Bediye S, Kehaliw A, Daba T, Getu KG, Assefa G (2010) Near Infrared Reflectance Spectroscopy (NIRS) for determination of chemical entities of natural pasture from Ethiopia. Agriculture and Biology Journal of North America 1: 919-922.

Fernández VM, Garrido AM, Dardenne P (2006) Evaluation of Pretreatment Strategies for Near-Infrared Spectroscopy Calibration Development of Unground and Ground Compound Feed in stuffs. Applied Spectroscopy 60: 17-23.

Freitas JC, Santos AS, Tomich TR, Franco GL (2016) Predição do valor nutritivo de gramínea nativa e exótica no pantanal por meio do método de reflectância no infravermelho próximo. Veterinária e Zootecnia 23: 251259.

García, E (1987) Modificaciones al sistema de clasificación climática de Köppen. Instituto de geografía Universidad Nacional Autónoma de México. México. 98p.

Gonçalves de LJ, Fernandes FMA, de Sousa TR, Dos Santos FS (2018) Utilização do NIRS na determinação dos parâmetros de digestibilidade e consumo em ruminantes. Nutri Time 15: 1-13.

Hall MB, Hoover WH, Jennings JP, Webster TKM (1999) A method for partitioning neutral detergent-soluble carbohydrates. Journal of the Science of Food and Agriculture 79: 2079-2086.

Hernández EA, Juárez LFI, Pell AN, Montero LM, Pinos RJM, Blake RW (2020) Degradación ruminal in vitro de las fracciones de carbohidratos contenidas en pastos tropicales fertilizados con nitrógeno. Revista Mexicana de Ciencias Pecuarias 11: 266-282.

Lanzas C, Sniffen CJ, Seo S, Tedeschi LO, Fox DG (2007) A revised CNCPS feed carbohydrate fractionation scheme for formulating rations for ruminants. Animal Feed Science Technology 136: 167-190.

Li J, Li Q, Li D, Chen Y, Wang X, Yang W, Zhang L (2016) Use of near-infrared reflectance spectroscopy for the rapid determination of the digestible energy and metabolizable energy content of corn fed to growing pigs. Journal of Animal Science and Biotechnology 7: 1-9.

Licitra G, Hernandez TM, Van Soest PJ (1996) Standardization of procedures for nitrogen fractionation of ruminant feeds. Animal Feed Science and Technology 57: 347-358.

Lobos I, Gou P, Hube S, Saldana R, Alfaro M (2013) Evaluation of potential NIRS to predict pastures nutritive value. Journal of Soil Science and Plant Nutrition 13: 463-468. 
Lobos I, Moscoso CJ, Pavez P (2019) Modelos de calibración para la calidad nutricional de pastos frescos por espectroscopia de reflectancia de infrarrojo cercano. Ciencia e Investigación Agraria 46: 234-242.

Mazabel J, Worthington M, Castiblanco V, Peters M, Arango J (2020) Using near infrared reflectance spectroscopy for estimating nutritional quality of Brachiaria humidicola in breeding selections. Agrosystem Geosciences \& Environment 3: e20070. DOI: 10.1002/agg2.20070.

Molano ML, Cortés ML, Ávila PÁ, Martens SD, Muñoz LS (2016) Ecuaciones de calibración en espectroscopía de reflectancia en el infrarrojo cercano (NIRS) para predicción de parámetros nutritivos en forrajes tropicales. Tropical Grasslands-Forrajes Tropicales 4: 139-145.

Monrroy M, Gutiérrez D, Miranda M, Hernández K, Renán GJ (2017) Determination of Brachiaria spp. forage quality by near-infrared spectroscopy and partial least squares regression. Journal of the Chilean Chemical Society 62: 3472-3477.

Nie Z, Tremblay GF, Bélanger G, Berthiaume R, Castonguay Y, Bertrand A, Michaud R, Allard G, Han J (2009) Near-infrared reflectance spectroscopy prediction of neutral detergent-soluble carbohydrates in timothy and alfalfa. Journal of Dairy Science 92: 1702-1711.

Parrini S, Acciaiol, A, Crovetti A, Bozzi R (2018) Use of FT-NIRS for determination of chemical components and nutritional value of natural pasture. Italian Journal of Animal Science 17: 87-91.

Pasquini C (2003) Near infrared spectroscopy: Fundamentals, practical aspects and analytical applications. Journal of the Brazilian Chemical Society 14: 198-219.

Piaskowski JL, Browun D, Campell KG (2016) Near-Infrared calibration of soluble stem carbohydrates for predicting drought toletance in spring wheat. Agronomy Journal 108: 285-293.

Ramirez JA, Posada JM, Handa IT, Günter H, Vohland M, Messier C, Björn R (2015) Near-infrared spectroscopy (NIRS) predicts non-structural carbohydrate concentrations in different tissue types of a broad range of tree species. Methods in Ecology and Evolution 6: 1018-1025.

Roberts CA, Workman J, Reeves JB (2003) Near infrared spectroscopy in agriculture. Agronomy 44. American Society of Agronomy, Inc. Madison, WI, USA. 785p.

Segura SF, Echeverri FR, Patiño LIAC, Mejía GAI (2007) Descripción y discusión acerca de los métodos de análisis de fibra y del valor nutricional de forrajes y alimentos para animales. VITAE Revista de la Facultad de Química Farmacéutica 14: 72-81.

Shenderey C, Shmulevich I, Alchanatis V, Egozi H, Hoffman A, Ostrovsky V, Lurie S, Ben AR, Schmilovitch Z (2010) NIRS detection of moldy core in apples. Food and Bioprocess Technology 3: 79-86.

Shenk JS, Westerhaus MO (1996) Calibration the ISI way. In: Davies AMC, Williams PC (eds). Near Infrared Spectroscopy: The future waves. NIR Publications, Chichester. UK. pp: 198-202.

Sniffen, CJ, O'connor JD, Van Soest PJ, Fox DG, Russell JB (1992) A net carbohydrate and protein system for evaluating cattle diets. II. Carbohydrate and protein availability. Journal of Animal Science 70: 3562-3577.

Stuth, J, Jama A, Tolleson D (2003) Direct and indirect means of predicting forage quality through near infrared reflectance spectroscopy. Field Crops Research 84: 45-56.

Valenciaga D, Saliba EOS (2006) La espectroscopia de reflectancia en el infrarrojo cercano (NIRS) y sus potencialidades para la evaluación de forrajes. Revista Cubana de Ciencia Agrícola 40: 259-267.

Van Soest PJ, Robertson JB, Lewis BA (1991) Methods for dietary fiber, neutral detergent fiber, and non-starch polysaccharides in relation to animal nutrition. Journal Dairy Science 74: 3583-3597. 
Van Soest PJ (1994) Nutritional ecology of the ruminant. 2nd ed. Cornell University Press. Ithaca, New York, USA. 476p.

Vásquez DR, Abadía B, Arreaza LC (2004) Aplicación de la espectroscopía de reflectancia en el infrarrojo cercano (NIRS) para la caracterización nutricional del pasto Guinea y del grano de maíz. Revista Corpoica 5 : 49-55.

Wilkins RJ (2000) Forages and their role in animal systems. In: Givens DI, Owen E, Axford RFE, Omed HM (ed). Forage evaluation in ruminant nutrition. CAB International. London, UK. pp: 1-14.

Williams PC (2004) Near-infrared technology - Getting the best out of light: A short course in the practical implementation of near-infrared spectroscopy for the user. Edition 2.0. PDK Projects Inc. Nanaimo, BC, Canadá. 311p.

Williams P (2014) The RPD statistic: A tutorial note. NIR News 25: 22-26. 